\title{
Handling immune-suppressive therapies during SARS-CoV-2 pandemic: insights from pediatric datasets
}

\author{
Andrea Angeletti ${ }^{1} \cdot$ Paolo Cravedi $^{2} \cdot$ Gian Marco Ghiggeri ${ }^{1}$
}

Published online: 24 September 2020

(C) Italian Society of Nephrology 2020

Coronavirus Disease 2019 (COVID-19) represents a global public health emergency [1]. At the onset of this pandemic, medical efforts were focused on treating affected patients and protecting others from infection. As the pandemic continues, physicians are dealing with the need to maintain hospital facilities at high levels of preparedness for urgent care of COVID-19 patients and, at the same time, to provide heath assistance to non-infected individuals. While SARS-Cov-2 is spreading worldwide, nephrologists have been facing the dilemma of continuing immunosuppression in patients with immune-mediated glomerulonephritis and in kidney transplant recipients, trying to balance the beneficial effects of immunosuppression in controlling auto- and allo-immune responses with the potential risks of inhibiting immune response against the virus [2].

SARS-Cov-2 infection has had a relevant impact, in terms of incidence and disease severity, on adult kidney transplant recipients. A study from Brescia, Lombardy, the Italian region with one of the highest rates of SARS-CoV-2 worldwide, reported an incidence of COVID-19 of $4.6 \%$ among a population of 1200 kidney transplant recipients and a mortality rate of $27 \%(15 / 55)$, which is higher than the rates reported in the general population [3]. A more recent survey, involving a population of 9845 kidney transplant recipients across 14 centers worldwide (TANGO consortium), confirmed the Italian data and reported an incidence

Electronic supplementary material The online version of this article (https://doi.org/10.1007/s40620-020-00821-1) contains supplementary material, which is available to authorized users.

Gian Marco Ghiggeri

gmarcoghiggeri@gaslini.org

1 Division of Nephrology, Dialysis, Transplantation, IRCCS Giannini Gaslini Children's Hospital, Via Gerolamo Gaslini 5, 16148 Genova, Italy

2 Department of Medicine, Translational Transplant Research Center, Icahn School of Medicine At Mount Sinai, New York, NY, USA of infection of $1.5 \%$, with a mortality rate of $32 \%(44 / 144)$ during a median follow-up of 52 days [4].

At variance, data on COVID-19 in patients on chronic immunosuppressive therapies for glomerulonephritis are scarce and even less clear in pediatric patients. The common feeling is to limit immunosuppressive therapy to patients at risk of progressive kidney injury [5]. The risk is to delay and even omit effective treatments for patients affected by chronic renal disease, usually characterized by slow but progressive evolution. Considering the risk of a second wave in countries where COVID-19 cases are now declining, or considering the emergencies in nations that are now facing the coronavirus, evidence-based recommendations are of need.

\section{COVID-19 in children with kidney disease}

Children seem to be at lower risk of SARS-CoV-2 infection and the positive cases have a milder course of COVID-19. In areas at high incidence of COVID-19, such as Bergamo, Italy, individuals $<14$ years of age accounted for $0.2 \%$ of total cases [6]. However, data on the incidence of COVID19 in children affected by kidney diseases on immunosuppressive treatments are limited. The European Rare Kidney Disease Reference Network (ERKnet) has described 18 pediatric kidney patients (median age 11.5 years) from 16 countries, with a diagnosis of COVID-19, on chronic treatment with steroids, tacrolimus or mycophenolate mofetil. Three of them had been previously treated with the antiCD20 monoclonal antibody rituximab. None of the patients required hospitalization in intensive care and seven were treated as outpatients [7]. As the SARS-CoV-2 pandemic spread worldwide, in the few months since the first report the ERKnet survey has been enriched with new cases to a total of 113 children with a diagnosis of COVID-19, 12 of whom had been treated with rituximab or ofatumumab within 6 months prior to the infection (Franz Schaefer, personal communication). Clinical outcomes of this survey 
are impatiently awaited for more defined conclusions. The ERKnet consortium involved thousands of patients, covering many areas outside Europe, including the U.S. and China, therefore the total number of children considered in the survey is still not known: lack of exact information prevents an estimation of COVID-19 incidence in children with chronic glomerulonephritis.

A plausible conclusion is that the impact of COVID-19 in this population is low and very few subjects who had previously been treated with anti-CD20 antibodies contracted SARS-CoV-2 infection. Confirming this observation would reinforce the idea of maintaining immunosuppressive approaches also in the era of the SARS-CoV-2 pandemic.

\section{Insights from large pediatric databases on nephrotic syndrome and kidney transplant}

Due to the relative rarity of these diseases, performing ad hoc prospective studies or creating dedicated registries represent a challenge during the COVID-19 pandemic. As a quick alternative strategy to get insights into this population, we decided to carry out comprehensive interviews with pediatric patients and young adults enrolled in a clinical study on nephrotic syndrome. We followed a similar strategy for pediatric kidney transplant recipients at our center.

From February 24 to April 7, 2020, all the patients included in the two studies coordinated by the Division of Nephrology, Dialysis and Transplantation of the Gaslini Children's Hospital, Genoa, Italy, underwent weekly questionnaires by phone, and from April 7 to July 15, 2020, all participants were requested to refer any clinical information suggesting possible infectious disease.

In the first study, we prospectively followed-up a cohort of 159 children (median age 12.5 years) with multi-drug dependent nephrotic syndrome who had received one or more infusions of anti-CD20 antibodies (rituximab or ofatumumab) within the prior 4 years, and one infusion within the 12 preceding months. All 159 patients had been previously enrolled in a single-center randomized controlled trial comparing the safety/efficacy profile of two B-celldepleting antibodies (NCT02394119) and represented the entire cohort of patients enrolled in the trial. Participation in a trial guarantees periodic controls and a relative homogeneity of basal clinical characteristics. In particular, our study involved patients from all Italian regions, with a predominance of areas with a high incidence of SARSCov-2 infection (Supplementary Fig. 1). At the beginning of the observation period, immune characteristics included, on average, low B cell levels, and low serum IgG and Natural Killers in approximately half of them. None of the patients reported symptoms of COVID-19. Six patients had household members affected by COVID-19, two of whom died of lung complications. Despite the close contact, none of the six subjects reported any symptoms related to COVID-19 and all resulted negative for the antiSARS-CoV-2 antibodies (IgM and $\operatorname{IgG}$ ) [8].

During the same period, we also prospectively followed-up 160 kidney transplant recipients, including 64 children ( $\leq 18$ years) and 96 young adults (19-30 years) who had received a renal transplant at our center over the last 10 years and were maintained on triple immunosuppressive therapy [9]. Similarly to the previous cohort, none of the 160 transplanted patients reported clinical symptoms of COVID-19. Two patients ( $\leq 18$ years) had household members who developed severe COVID-19 requiring hospitalization, but swab tests of both of our patients resulted negative for SARS-CoV-2. Immunosuppressive therapy was not modified and neither subject reported any symptoms [9].

\section{Potential impact on the international recommendations}

International recommendations on the management of immunosuppression in COVID-19 patients with glomerulonephritis or renal transplant are still limited and are based on few observations. Our reports are in line with the results of the ERKnet surveys and favor maintaining chronic immunosuppressive therapies both in children and young adults with immune-mediated glomerular diseases or kidney transplant. The absence of COVID-19 in children who received antiCD20 therapy is of particular interest: as an indirect side effect, this pandemic has reduced the treatments of patients with immunological diseases in need of long-acting immunosuppressive therapies, due to the supposed increased risk of contracting SARS-Cov-2 infection [2].

Based on our results, B-cell depleting therapies should be, in our opinion, considered safe in young patients with glomerular disease, supporting the idea that the administration of rituximab should not be restrained even in areas at high incidence of SARS-CoV-2, provided that adequate protection measures are followed.

Similarly, our data support the concept that anti-rejection therapy should not be modified in pediatric kidney transplant recipients, as the perceived (but not demonstrated) risk of infection may expose patients to an augmented risk of acute rejection. Similarly, the activity of pediatric renal transplantation could be safely continued during the COVID-19 pandemic.

Should these observations obtained in children impact the conservative strategies suggested for the treatment of glomerulonephritis in adults? 
It probably should, and similarly to what has been done in children, further evidence based on interview strategies on homogeneous cohorts of adults are needed.

In particular, our interview strategy, involving patients already enrolled in large databases, allowed to generate data in a relatively short time frame. We hope that the pediatric approaches could stimulate further research and allow a more evidence-based and effective handling of renal disease also in adults.

Acknowledgements Authors thank Prof Franz Schafer for providing details of the ERKnet survey. GMG was supported for the trial (NCT02394119) by a grant from the Italian Ministry of Health (RicercaFinalizzata2016:WFR: PE-2016-02361576). The Giannini Gaslini Institute provided logistic and financial support to the study through grants from the Italian Ministry of Health ('Cinque per mille of IRPEF-Finanziamento della ricerca sanitaria'). People working on the project on therapeutic approaches to nephrotic syndrome are employed by the "Fondazione Malattie Renali del Bambino" whose financial support we acknowledge. Grant ROL 9849 was received from Compagnia di San Paolo.

\section{Compliance with ethical standards}

Conflict of interest Authors declare no conflicts of interest.

Ethical approval This article does not contain any studies with human participants or animals performed by any of the authors.

Informed consent For this type of study formal consent is not required.

\section{References}

1. Zhu N, Zhang D, Wang W, Li X, Yang B, Song J et al (2020) A novel coronavirus from patients with pneumonia in China, 2019. N Engl J Med 382(8):727-733
2. Kronbichler A, Gauckler P, Windpessl M, Il Shin J, Jha V, Rovin BH et al (2020) COVID-19: implications for immunosuppression in kidney disease and transplantation. Nat Rev Nephrol 16(7):365-367

3. Bossini N, Alberici F, Delbarba E, Valerio F, Manenti C, Possenti S et al (2020) Kidney transplant patients with SARS-CoV-2 infection: the brescia renal COVID task force experience. Am J Transplant. https://doi.org/10.1111/ajt.16176

4. Cravedi P, Suraj SM, Azzi Y, Haverly M, Farouk S, Perez-Saez MJ et al (2020) COVID-19 and kidney transplantation: results from the TANGO international transplant consortium. Am J Transplant. https://doi.org/10.1111/ajt.16185

5. Bomback AS, Canetta PA, Ahn W, Ahmad SB, Radhakrishnan J, Appel GB (2020) How COVID-19 has changed the management of glomerular diseases. Clin J Am Soc Nephrol 15(6):876-879. https://doi.org/10.2215/CJN.04530420

6. Grasselli G, Pesenti A, Cecconi M (2020) Critical care utilization for the COVID-19 outbreak in Lombardy, Italy: early experience and forecast during an emergency response. JAMA. https://doi. org/10.1001/jama.2020.4031

7. Marlais M, Wlodkowski T, Vivarelli M, Pape L, Tonshoff B, Schaefer F et al (2020) The severity of COVID-19 in children on immunosuppressive medication. Lancet Child Adolesc Health 4(7):e17-e18

8. Angeletti A, Drovandi S, Sanguineri F, Santaniello M, Ferrando G, Forno R, Cipresso G, Caridi G, Riella LV, Cravedi P, Ghiggeri GM (2020) COVID-19 in children with nephrotic syndrome on Anti-CD20 chronic immunosuppression. Clin J Am Soc Nephrol. https://doi.org/10.2215/CJN.06400420

9. Angeletti A, Trivelli A, Magnasco A, Drovandi S, Sanguineri F, Santaniello M et al (2020) Risk of COVID-19 in young kidney transplant recipients. Results from a single-center observational study. Clin Transplant 34:e13889. https://doi.org/10.1111/ ctr. 13889

Publisher's Note Springer Nature remains neutral with regard to jurisdictional claims in published maps and institutional affiliations. 